\title{
Advancements in research and development to combat COVID-19 using nanotechnology
}

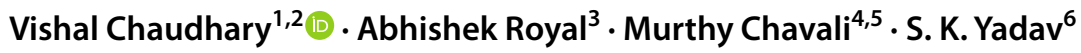

Received: 7 July 2020 / Accepted: 5 January 2021 / Published online: 25 January 2021

(c) The Author(s), under exclusive licence to Springer Nature Switzerland AG part of Springer Nature 2021

\begin{abstract}
The whole world is currently facing a global health crisis due to the coronavirus disease (COVID-19) pandemic caused by SARS coronavirus 2, which started in Wuhan City, China, in December 2019. The pandemic has affected 235 countries, areas or territories and infected over 42 million people across the globe as per WHO update on 27 October 2020. More than 1.1 million people have died and the numbers are increasing daily. However, some drugs have been authorized for emergency treatment of patients, medication and vaccines with proven efficacy to prevent and treat the disease is still under various phases of development. The entire world is consistently making efforts to address three major challenges related to COVID19 including prevention of its spread, prompt and early diagnosis and treatment of patients to save lives. Touted as one of the game-changing technologies of the century, nanotechnology has huge potential to develop solutions against these three major challenges of the disease. Nanotechnology comprises of multidisciplinary prospects encompassing diverse disciplines including medicine, material science, artificial intelligence, environment, virology, physical sciences, chemistry and biology. The numerous challenges can be addressed through the engineering of the various physicochemical properties of materials presents in abundance in nature. Various claims, studies and reports on research and development to combat these challenges associated with COVID-19 have been collectively discussed in this article from the perspectives of nanotechnology.
\end{abstract}

Keywords COVID-19 $\cdot$ Nanotechnology $\cdot$ Nanomaterials $\cdot$ Biosensors $\cdot$ Graphene

\section{Introduction}

The outbreak of coronavirus disease (COVID-19) caused due to severe acute respiratory syndrome coronavirus 2 (SARS-CoV-2) has become a foremost global health concern [1]. The size of this virus is in nanoscale dimensions,

Vishal Chaudhary

chaudhary00vishal@gmail.com

1 Research Cell and Department of Physics, Bhagini Nivedita College, University of Delhi, Delhi, India

2 Department of Physics and Astrophysics, University of Delhi, Delhi, India

3 Faculty of Medicine, Public Health and Nursing, Universitas Gadjah Mada, Yogyakarta, Indonesia

4 PG Department of Chemistry, Dharma Appa Rao College (DARC), Nuzvid Krishna 521201, Andhra Pradesh, India

5 NTRC-MCETRC and Aarshanano Composite Technologies Pvt. Ltd, Guntur District, Andhra Pradesh, India

6 Department of Physics, Shivaji College, University of Delhi, Delhi, India ranging from $65-125 \mathrm{~nm}$ in diameter [2]. The outbreak has been declared as a pandemic by the World Health Organization (WHO) on 11 March 2020, and it has currently affected more than 235 countries, areas and regions worldwide [3]. According to the current pieces of evidence, the virus gets transmitted through the exposure of mucosal surfaces with infected respiratory droplets (of diameter $>5-10 \mu \mathrm{m}$ ) and contact with contaminated surfaces [4]. Airborne transmission may be possible in specific settings where aerosols are generated during procedures or supportive treatment leading to the suspension of droplet nuclei (of diameter $<5 \mu \mathrm{m}$ ) containing virus for longer periods in the air [4]. Therefore, direct contact with the droplets of infected people released while coughing or sneezing within the proximity of $1 \mathrm{~m}$, contact with the contaminated surfaces (objects used on/by patients) and indirect contact with infective aerosols which can remain suspended in the air for a longer time and at distance greater than $1 \mathrm{~m}$ are the possible routes of transmission [4]. There is documented evidence of intestinal infection and presence of this virus in faeces [5], but faeco-oral route of transmission has not been reported till date. 
The three major challenges associated with COVID-19 include its prevention, prompt and early diagnosis and treatment. At present, there are no approved drugs and vaccines to combat COVID-19. However, there are various claims worldwide about the development of vaccines and drugs under various phases of clinical trials [6-10]. Currently available therapies include use of non-specific antivirals and symptomatic treatment to reduce the severity of the disease, hospital stay and save lives [6-10]. Thus, preventive measures remain the main strategy to control the transmission of this infection which includes dedicated use of masks, social (physical) distancing, use of face shields (when social distancing cannot be maintained) and practising hand hygiene [6-10]. Determining the outbreak at an early stage is the major concern of researchers, which has led to enormous efforts in the development of early diagnostic measures including advanced rapid test, artificial intelligence-based pre-patterned tests and computed tomography (CT) scans [6-10]. Various forthcoming measures of prevention, detection and cure have been researched and enhanced using nanotechnology [6-10]. Therefore, continuous research and development to enhance the characteristics of these preventive, diagnostic and treatment modalities through the application of nanotechnology is the prime focus of the scientific community [14-16]. Nanotechnology is the branch of science and technology that deals with research and development conducted at dimensions of less than $100 \mathrm{~nm}$. The materials ranging in this dimensional scale $(1-100 \mathrm{~nm})$ are termed as nanomaterials [17]. A striking characteristic of nanostructures is the changes in their properties with size [17]. Such changes in properties begin to occur when the size approaches a characteristic scaling length which is usually different for each property or phenomenon [17]. Nanomaterials possess enhanced physical and chemical properties as compared to their bulk counterparts such as reactivity, colour, melting point, hydrophobicity and ductility [17]. The reason behind the enhancement of their physical and chemical properties lies in "size effects" which include surface effects and quantum size effects [17]. With the reduction in the size of materials, its surface area-to-volume ratio increases; this enhances its surface reactivity and known as surface effects [17]. However, the enhancements in chemical properties such as alteration in bandgap are due to quantum size effects due to the very small size of particles [17]. These enhanced properties with reduced size have made nanomaterials as a special candidate and attracted the subordinate amount of attention of researchers in every field [17]. Nanomaterials possess a wide range of applications in diversified fields including medicines, sensors, actuators, defence, electronics, water and air purification, drug delivery, cosmetics, aero sciences, energy production and storage, food and beverages, virology, textiles, automobiles, agriculture, communication system and many more [14-20].
There is increasing attention towards the production of newgeneration low-cost, energy-efficient, portable and environment and user-friendly smart devices using nanotechnology [14-20]. Thus, the potential of applications of nanotechnology to address various global concerns including pandemics, pollution, ozone depletion, global warming, renewable and clean energy production, and waste management is enormous and under exploration [14-20].

Nanotechnology has already played a vital role in inhibiting various viruses with the action of different nanomaterials including graphene oxide, carbon nanomaterials, metal nanoparticles, silica nanoparticles and nanoclay as illustrated in Fig. 1. There are various interactions of these nanoparticles take place with virus components including electrostatic interaction, reactive oxygen generated species type interaction, dative bond and hydrophobic interaction [21]. Table 1 illustrates the various nanomaterials used to inhibit different viruses. However, the inhibition or detection can also be done by using nanomaterials as external stimulus such as in the case of biosensors [21]. The various components of virus conjugate with nanomaterials and present as an external stimulus, which results in the generation of monitoring or detection signals [21. Such conjugation between virus genome and nanomaterials is illustrated later in "Biosensors" section of this article [21].

Since the size of coronaviruses lies in nanoscale dimensions, nanotechnology possesses an enormous potential to understand the structure, growth and life span of this virus [2, 14-18]. Nanotechnology can bring new prospects in the development of affordable and scalable detection methods, safe personal protective equipment and new effective medical solutions. The following sections will describe the role and potential of nanotechnology to address all three major aspects of COVID-19 pandemic.

\section{Understanding COVID-19 through nanotechnology: structure and life cycle}

The development of cell culture systems and electron microscope has played a significant role in the identification and isolation of new viruses [14-16]. The invention of immunoassays and polymerase chain reaction (PCR) techniques has revolutionized the field of diagnostic virology [10-13]. The unique properties of nanomaterials have been effectively exploited in the rapid development of a wide range of serological and molecular detection techniques for isolation of new viruses [14-22]. These nanomaterial-based technologies were extensively used to identify and understand SARS (severe acute respiratory syndrome) virus and MERS (Middle East respiratory syndrome) virus, which are close relatives of SARS-CoV-2 
Fig. 1 Various nanomaterials used to inhibit different viruses

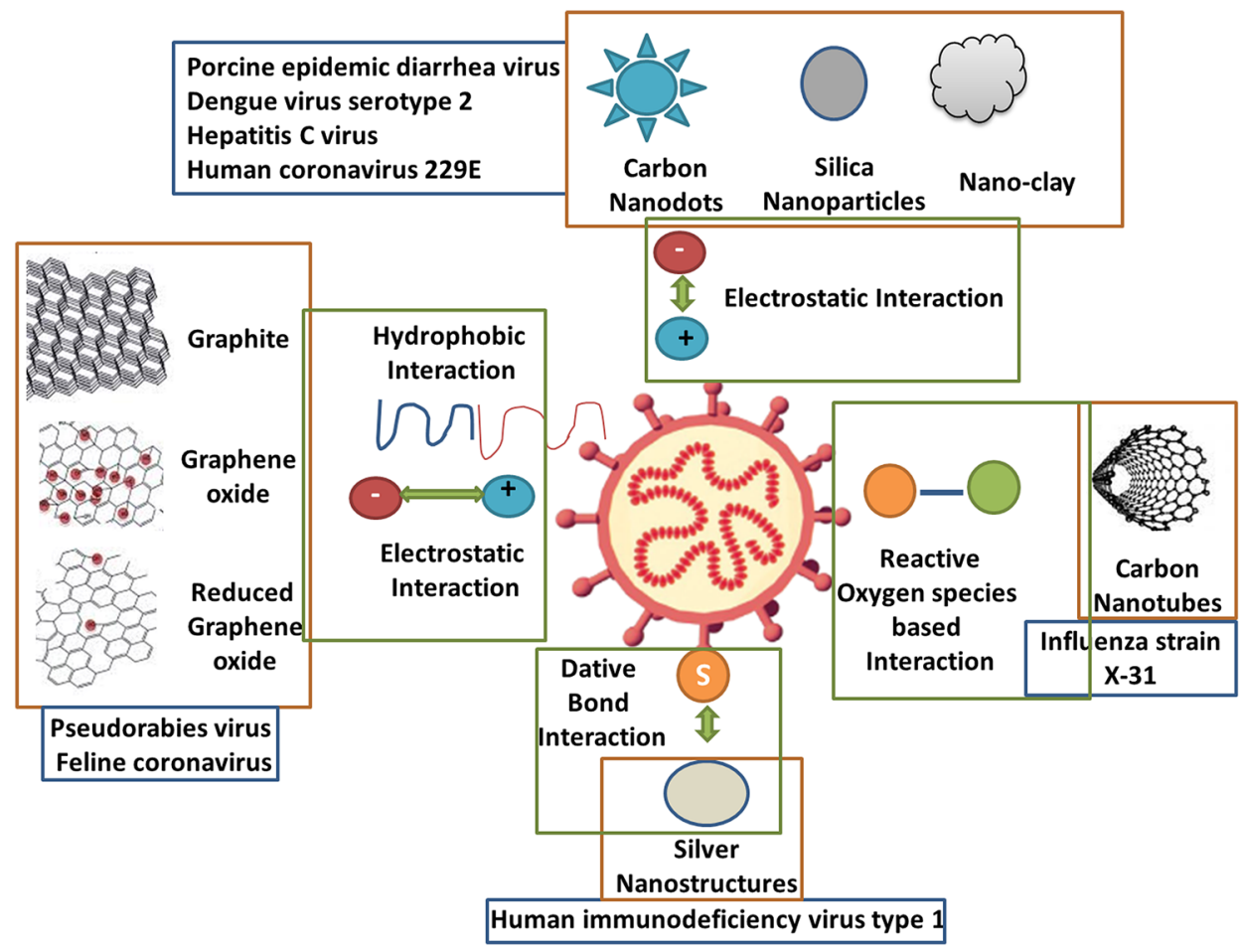

and were responsible for regional outbreaks in the twentyfirst century and are currently being used to understand the structure and life cycle of SARS-CoV-2 [14-16, 21, 22]

SARS-CoV-2 is an enveloped, non-segmented, singlestranded positive-sense RNA virus having pikes on the outer surface as illustrated in various studies [14-23]. It possesses four main structural proteins: spike $(S)$ glycoprotein, membrane $(M)$ glycoprotein, envelope $(E)$ glycoprotein and nucleocapsid $(\mathrm{N})$ protein [22]. The genome of SARS-CoV-2 shows similarities with other coronaviruses [22]. The main target of this virus is angiotensin-converting enzyme II (ACE2) receptors present on the surface of host cells [21-23]. The C-terminal domain of SARS$\mathrm{CoV}-2$ consisting of envelop-embedded spike $(S)$ protein binds to ACE2 receptor of the host cell surface [23]. By solving the crystal structure of the complex produced, the molecular details of this interaction can be illustrated. Since the first stage of infection consists of binding this $S$ protein to ACE2 receptor, the treatment approaches to target this linking/ binding event [23]. Figure 2 illustrates the binding event of S protein of SARS-CoV-2 to ACE2 receptor. The entry of virus into cell is facilitated by transmembrane serine protease 2 (TMPRSS2) through protease activity [23]. Following internalization, the virus particles enter endosomes. Since the $\mathrm{pH}$ of endosomes is low, the uncoated viral particle releases genome for protein synthesis [23]. This results in syntheses of viral RNA and protein, which creates new infectious particles and release them in the host cell [21-23].

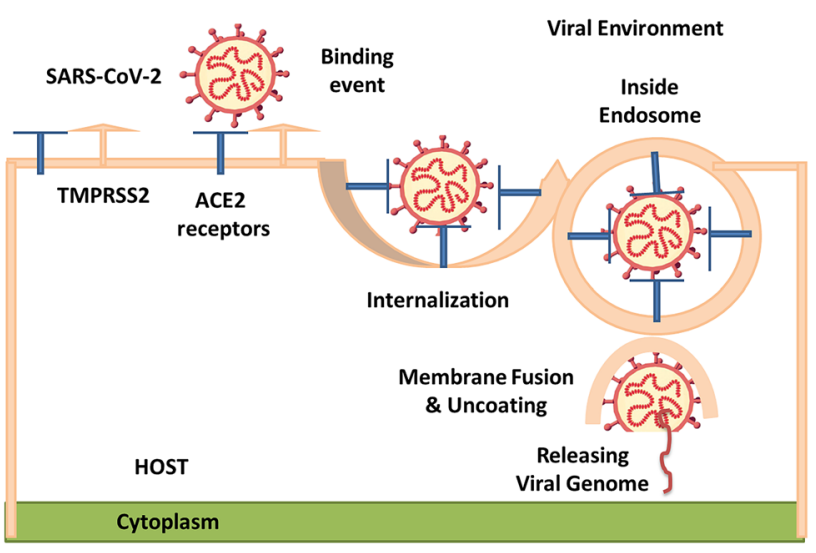

Fig. 2 Interaction of SARS-CoV-2 with host cell

\section{Prevention}

Considering the various modes of transmission of COVID19 through cough, sneeze and other biofluids, the first approach to combat this virus is to prevent its propagation through contaminated air, skin or surfaces [14-16]. Though there is the availability of a range of preventive tools in the market, widespread research and development is still taking place in light of nanotechnology to enhance their preventive capabilities in terms of less toxicity, no adverse effects, high sterilization capability with a low dosage, longer lifetime and more environment- and user-friendly [14-26]. 
Table 1 Nanomaterials used for inhibiting various viruses [21]

\begin{tabular}{llll}
\hline S. no & Nanomaterials used & Target viruses & Type of interaction \\
\hline 1 & Graphene oxide, graphite, reduced graphene oxide & $\begin{array}{l}\text { Pseudorabies virus feline } \\
\text { Coronavirus }\end{array}$ & Electrostatic and hydrophobic \\
2 & Carbon nanodots, silica nanoparticles, nanoclay and its & $\begin{array}{l}\text { Porcine epidemic diarrhoea virus } \\
\text { composites }\end{array}$ & $\begin{array}{l}\text { Dengue virus serotype 2 } \\
\text { Electrostatic }\end{array}$ \\
& & Hepatitis C virus & Human coronavirus 229E \\
& Silver nanoparticle & Human immunodeficiency virus type 1 & Dative bond \\
4 & Multiwall carbon nanotubes & Influenza strain X-31 & Reactive oxygen species \\
\hline
\end{tabular}

\section{Vaccine}

Vaccines are biological preparations that stimulate the body's immune system to recognize their antigen as a foreign agent and develop antibodies and memory cells to fight against it. These cells initiate a cascade leading to the destruction of the micro-organism having a similar antigen, thereby preventing the infection in future encounters [22-24]. The multifunctional nature of nanoparticle-based vaccines can be tailored to address the unique challenges posed by the broad array of micro-organisms $[23,24]$. The nanoparticles can be used to activate cellular immunity and/ or potentiate immune response with adjuvants [24]. Currently, 38 candidate vaccines are in the phase of clinical evaluation and about 149 candidate vaccines are in pre-clinical evaluation for COVID-19 around the world (Table 2) [23-29]. These potential vaccines are using particles/ antigens ranging from live attenuated to an inactivated virus, DNA, RNA, mRNA, replicating as well as non-replicating viral vector and protein subunits [24-29].

In mRNA-based technologies, nanoparticle-based drug release approaches have been commonly used. In these techniques, a part of RNA required for preparation of vaccine is synthesized and embedded in lipid nanoparticles (LNPs). However, in the self-replicating RNA-based therapeutic vaccines, a leading nanoparticle non-viral delivery system (LUNAR) has been used to produce proteins inside the body [24-29]. In vector-based vaccines, an adenovirus as a nanoviral vector has been used to deliver the vaccine agent [24-29]. Protein subunit-based vaccines consist of designing the recombinant $\mathrm{F}$-proteins to self-assemble into nanoparticle constructs [24-29]. However, DNAbased vaccines consist of using neutral lipid formulation (liposome-based) with the high efficacy of the fusogenic protein-mediated delivery technology. The virus-like particle (VLP) vaccines have been designed using VLPs to mimic the native structure of viruses, allowing them to be easily recognized by the immune system [29]. Thus, intensive research is going on to develop and testing a successful vaccine based on nanotechnology at various clinical stages [24-29].

Table 2 COVID-19 Candidate Vaccines in Clinical Evaluation (22 September 2020) [23-29]

\begin{tabular}{|c|c|c|c|}
\hline Vaccine platform & Types of candidate vaccines & $\begin{array}{l}\text { Number of } \\
\text { candidates }\end{array}$ & Example \\
\hline Non-replicating viral vector & $\begin{array}{l}\text { ChAdOx1-S, adenovirus type } 5 \text { vector, adeno-based (rAd26-S + rAd5- } \\
\text { S), Ad26COVS1, replication defective simian adenovirus (GRAd) } \\
\text { encoding S, Ad5-nCoV }\end{array}$ & 6 & AstraZeneca, ReiThera \\
\hline Replicating viral vector & Measle vector-based, intranasal flu-based RBD & 2 & Themis, Xiamen \\
\hline Inactivated & Inactivated, whole virion inactivated & 6 & Sinovac, Bharat Biotech \\
\hline RNA & $\begin{array}{l}\text { LNP-encapsulated mRNA, } 3 \text { LNP-mRNAs, mRNA, LNP-nCoVs- } \\
\text { aRNA }\end{array}$ & 6 & Moderna, Curevac \\
\hline Protein subunit & $\begin{array}{l}\text { Full-length recombinant SARS-CoV-2 glycoprotein nanoparticle } \\
\text { vaccine adjuvanted with matrix M, adjuvanted recombinant protein } \\
\text { (RBD-Dimer), RBD-based, S protein (baculovirus production }\end{array}$ & 13 & Novavax, Covaxx \\
\hline DNA & $\begin{array}{l}\text { DNA plasmid vaccine with electroporation, DNA plasmid vac- } \\
\text { cine + Adjuvant, DNA plasmid vaccine, DNA vaccine (GX-19) }\end{array}$ & 4 & Cadila, Genexine Consortium \\
\hline VLP & Plant-derived VLP adjuvanted with GSK or dynavax adjs & 1 & Medicago Inc \\
\hline
\end{tabular}




\section{Sanitizers and disinfectants}

Disinfectants and sanitizers available in the market are majorly based on alcohol and its derivatives. These disinfectants offer protection to the user for a very short period ranging from few second to minutes [14-16, 30]. This disadvantage is due to the quick evaporation of volatile alcohol molecules from the user's skin. Subsequently, on coming into contact with a contaminated surface, the user again starts carrying the microbes. Further, these disinfectants being corrosive in nature are neither user- nor eco-friendly [14-16, 30-32]. Such disinfectants may affect human or animal health or their safety causing irritation in eyes and skin, leading to allergies and inflammation. They are also flammable due to the presence of alcohol and hydrogen peroxide possessing continuous threat of catching fire. The following section will describe several research reports in which nanotechnology has been used to address these shortcomings $[14-16,30]$.

\section{Metal nanoparticle-based disinfecting agents}

Metal nanoparticles (MNPs) such as silver, copper and titanium owing to their antibacterial and antiviral properties possess diverse applications in a variety of fields including medical, cosmetics and disinfectant productions [30-33]. The introduction of the nucleic acid of the virus into host cell causes viral infection, which is followed by its replication. Metal nanoparticles are believed to act on the surface of the virus and physically inhibit the interaction between the virus and host cells $[31,32]$. Contrary to alcohol-based disinfectants, MNPs such as silver and copper nanoparticles are environment- and user-friendly, non-inflammable and non-volatile, and can be fabricated using green technologies $[31,32]$. Therefore, MNP-based disinfectants and sanitizers possess enhanced characteristics in terms of fabrication, safety, health and life span to protect against COVID-19.

A non-foaming, non-irritating and eco-friendly nanosilver-based multipurpose sanitizer (NSMS) has also been developed by SHEPROS SDN BHD using a nanocolloidal technique with promising antiviral, antimicrobial and antifungal properties [29]. NanoTechSurface, Italy, has developed a sterilizing solution comprising of silver ions and titanium dioxide to disinfectant the coronavirus contaminated surface [30]. The adverse effects due to the toxicity of some MNPs on user health and environment can be prohibited by using biodegradable nanomaterials such as lipids or polymers [30]. As per the report published in Nature India [33], a Pune-based startup Weinnovate Biosolutions, sponsored by Department of Science and Technology (DST) and Department of Biotechnology (DBT), Government of India, has developed silver nanoparticle solution-based disinfectants and sanitizers, which are yet to be commercialized [33].
Naturally derived nanomaterials as adsorbents in disinfectants

Nanomaterials may also be fabricated using various green techniques including extraction from natural resources such as leaves, fruits, flowers, insects and animals [34]. These naturally extracted nanomaterials are eco-friendly, non-toxic, non-corrosive, user-friendly and cost-effective [34]. Owing to their larger effective surface area, these nanomaterials act as adsorbents in sanitizers and releases the disinfectant molecules in a controlled manner. Furthermore, such a larger effective surface area enhances the protective capabilities of disinfectants based on these nanomaterials due to larger interaction of disinfectant molecules with viruses present at the surface [14-16, 34].

As per a report in Times of India [35], Tamil Nadu Agricultural University has developed a naturally derived nanomaterial-based disinfectants, which is currently under patent process. According to their claims, the sanitizer can act up to 20-25 min, since the naturally derived nanomaterial acts as adsorbent and releases alcohol and hydrogen peroxide molecules in a steady and sustained manner [35]. Defence Institute of Advanced Technologies (DIAT) has also reported the fabrication of a disinfectant based on water spray using nanotechnology and named it as "Ananya" [36]. The disinfectant has been claimed to be effective for more than $24 \mathrm{~h}$ [36]. However, the formulation and constituents of "Ananya" disinfectant have not been disclosed yet. The nanotechnology-based disinfectant spray has been claimed to adhere to plastic, fabric and metallic objects very effectively [36]. Since it is a waterbased disinfectant, it is non-toxic, user- and eco-friendly with a shelf life of more than 6 months [36].

\section{Nanopolymer-based disinfectants}

Nanopolymer-based disinfectants such as PolyHMG (polyhexamethylene guanidine) polymer possessing antimicrobial properties have a greater effective life span and are easy to fabricate, cost-effective, non-inflammable, user- and eco-friendly, non-corrosive and biodegradable [37]. Wero Water Services has developed a disinfectant based on PolyHMG, which has been used by Prague public transit company from the Czech Republic for disinfecting their buses and vehicles [29, 38]. Design.123 has also developed a product PRELYNX PORTAL for scanning and sanitizing COVID-19. The portal sprays nanopolymer-based disinfectant vapours which inhibit most of the lipophilic and hydrophilic viruses present on the surface within 20 min of contact [29]. 


\section{Antiviral and antimicrobial nanolayers}

Various disinfectant coatings based on nanomaterials have been used as a major preventive measure against many viruses and bacteria [39]. Owing to their enhanced antiviral, antibacterial and antifungal properties, and higher effective surface area, these coatings are playing a significant role in the prevention of various infectious health hazards [39]. NANO4LIFE EUROPE L.P. has reported the development of a surface charge-based sanitizer, which inhibits and kills microbes based on physical charge effect. Sanitizer molecules form a positively charged layer termed as "swords" over the user surface [40]. These swords disrupt the negatively charged membrane of microbes and result in their death. The enhanced rapid action of these sanitizer molecules over microbes can be attributed to the use of nanomaterials owing to the coloumbic charge activity due to quantum size effects and their high effective surface area $[29,40]$.

\section{Light-Activated nanocoating disinfecting viruses}

Light-activated nanocoatings are made up of mineral nanocrystals that are charged by light of the visible range of spectrum including incandescent, fluorescent, light-emitting diodes (LED) and sunlight [41-43]. The interaction of these nanocrystals generates a powerful oxidation reaction which completely breaks down every organic contaminant present at the surface. Generally, these coatings are made up of nanoclusters of chemically modified metals such as gold embedded in a polymer matrix with a dye (such as crystal violet and methylene blue) possessing antimicrobial and antiviral properties [41-43]. On illumination to visible light, these dyes create reactive oxygen species, which damages the protective membrane and genetic material of viruses and kill them. This antiviral action of dye has been further enhanced by synthesizing their nanocomposites with metals including gold, zinc and silver using polymer matrix. These low-intensity visible light-activated coatings are superior in terms of safety as compared to other coatings based on UV light or high-intensity visible light [41-43]. Hwang et al. reported use of crystal violet dye with gold nanoclusters for effective killing of microbes with the application of low flux levels of white light. NanoTouch Materials, LLC, has also reported the development of a nanocoating using green nanotechnology based on mineral nanoparticles, which kills viruses by strong oxidation reaction at the surface after getting charged due to illumination of visible light [29-44].

\section{Nanoliposome technology-based disinfectants}

Nanoliposomes (size $60-80 \mathrm{~nm}$ ) are made up of naturally occurring lipids found in the cells of human, plants and animals [45]. These nanoliposomes possess antiviral and antimicrobial properties and inhibit the life cycle of the virus and bacteria. These naturally derived liposomes have been extensively used in shampoos, soaps and detergents owing to their antimicrobial and antiviral properties [45]. Many companies including Aneethun Professional, NANOMAX International, Richee Professional, LiQWD Professional Products, Nanogen, NanoDerm Pro, Kaedo Group, SHEPROS SDN. BHD and Suave Fragrance have used nanoliposomes for their disinfectant activity [29].

\section{Nanoelectrodes for disinfecting groundwater}

Researchers from Bar-Ilan University have reported developing an eco-friendly technique to disinfect viruses, bacteria and fungi based on an array of nanometer-shaped electrodes with enhanced surface properties. The meeting of water and electrodes produces a cleaning material in a unique aquatic environment [47]. The combination of these compounds gives rise to an effective microbicidal proficiency against bacteria, virus and fungi [29, 46, 47].

Thus, nanomaterials due to high effective surface area and antiviral properties have been extensively researched for disinfectants and possess huge potential for battle against COVID-19.

\section{Masks and PPE kits}

One of the most proficient preventive measures to protect from COVID-19 is to cover the face with mask [1, 4, 14, 14-16, 48]. It is essential to cover faces for both infected and non-infected individuals. An infected person can prevent the spread of virus produced during coughing or sneezing by covering his face, and a non-infected person can protect himself by covering face from the virus present in the air as droplets or aerosols, objects or accidental touching of mucus membranes. Masks are required to protect from airborne or touch-based transmission of the virus [1, 4, 14, 14-16, 29]. Face masks most commonly used filter out up to 95 percent of fine pollutants that are at least $300 \mathrm{~nm}$ in diameter and are referred to as N95 masks [14-16, 29, 48]. Though any kind of face cover can effectively prevent an individual from getting infected, but that mask itself becomes fomite since this virus have the capability to retain over the textile surface. Long-term use of masks also causes breathing issues, which can lead to the reduction of oxygen levels in the body. Largescale use of masks also causes concerns related to disposing off the masks. Another protective measure used by healthcare frontline workers is personal protective equipment (PPE) [1, 14-16, 49]. In general, PPE kits are not antiviral and antibacterial. However, the use of antiviral nanocoating and new nano-based material in PPE can enhance its protective capabilities [49]. 
Thus, to increase the protective efficiency of masks and other PPE and to address various related issues, various reports on nanotechnology-based innovations have been discussed in a further section [14-16, 48, 49].

\section{Nanoparticle technology}

Nanoparticles of various metals and metal oxides including silver, gold and copper owing to their highly effective surface area and microbicidal properties possess huge potential in the fabrication of antiviral coatings for face masks and PPE. A. K. Aggarwal et al. have reported developing an N9 nanosilver-based coating under Nanomission of DST, Government of India [50]. They are using this nanosilver-based antiviral coating for the fabrication of triple-layer medical mask in association with Resils Chemicals and Nanoclean Global Pvt. Ltd. IIT Guwahati, India, has also reported developing a spray based on copper and silver nanoparticles based on their antiviral properties to be used in face masks and PPE [51]. Promethean Particles Ltd has developed nanocopper particles which can be employed in face masks and PPE due to their antiviral and antimicrobial properties [52]. They have collaborated with textile companies to develop fabrics for healthcare sector based on novel copper nanoparticles embedded in a polymer matrix such as nylon to enhance their antiviral and antimicrobial properties. Sonovia Ltd. has developed an anti-pathogen fabric based on zinc oxide nanoparticles, which is washable and design effective, has antiviral properties and can be reused [29, 52]. This fabricated fabric has been used to make antiviral mask called Sonomasks and have potential to be used in PPE. G. Parks et al. have developed an antiviral coating based on a nanomaterial, which will capture the virus and then trigger a chemical reaction using ultraviolet light to destroy it [54]. Master Dynamic Limited has been working on developing antiviral coating based on nanodiamond, which can be further used in masks, PPE and other medical equipment including ventilators. The coatings are claimed to be of high performance, breathable and waterproof and possess antibacterial and antiviral properties that can block off, inhibit and even kill viruses [55]. X.TiO2 Inc. (XTI) has claimed to develop the world's first self-regenerating germicidal $\mathrm{TiO} 2 \mathrm{Ag}$ facemask based on XTI's revolutionary active nucleus nanoparticle technology. The germ-killing efficiency under zero light conditions has been claimed to be $99.999 \%$ [56]. The outer $\mathrm{TiO} 2$ layer of these masks refreshes itself in exposure to direct sunlight, thereby killing off all accumulated germs. These facemasks can offer significant protection as the first-hand protection against air-transmitted microbes including bacteria and viruses $[29,56]$.

\section{Nanofibrous membrane-based masks and PPE Kits}

A dense weblike network of nanofibres collectively forms nanofibrous membrane possessing high effective surface area. These nanofibrous membranes are incorporated into respiratory masks, providing high breathing and filtration efficiency. T. Rainey et al. have claimed to develop a biodegradable mask depending upon nanocellulose nanofibres, which can protect from contaminants of size up to $100 \mathrm{~nm}$ [57]. The nanocellulose nanofibres have been extracted from waste plant material including sugar cane bagasse and other agricultural waste. Kim et al. [58] have claimed to develop orthogonally aligned nanofibre $(\sim 100-500 \mathrm{~nm})$-based filter masks. These nanofibres have been fabricated using insulation block electrospinning technique. The orthogonally aligned structures minimize the pressure towards the air filter and maximize filtration efficiency. This nanofibre design is water-resistant, possess high filtering efficiency (94\%) and non-deformable (despite the 20 hand washes). YAMASHINFILTER CORP. has claimed to develop a nanoresin based on the nanofibrous mask inner sheets using synthetic polymers [59]. Owing to its unique properties including high polarity, larger effective surface area, self-extinguishing and heat or sound insulating properties, nanoresin has been used to make 3-D masks. Amrita Centre for Nanosciences and Molecular Medicine (ACNSMM) has claimed to develop cost-effective disposable masks based on nanofibrous technology [60]. These nanofibre-based masks possess two biodegradable and biocompatible layers of cloth. Metamasks have developed face masks made up of nanococo-carbon fibres (derivative of coconut shell and carbon) with a very thin membrane (less than $1 \mathrm{~nm}$ ), which can resist up to $99.99 \%$ of toxic airborne pollution from entering the body [61]. Nanopoli, Korea, has claimed to develop a nanofibre mask with air filtration rate around $98.75 \%$. These masks possess various layers including a water-repellent layer made of non-woven fabric and skin-friendly silk layer [29, 62].

\section{Graphene and its derivative-based masks and PPE kits}

Graphene is a two-dimensional hexagonal arrangement of single-carbon atoms, whereas graphene oxide (GO) is its oxidized equivalent [63]. GO can be reduced to reduced GO (rGO) by removing oxygen groups with the action of reducing agents. These materials are single-layer materials possessing high effective surface area and high absorbing capability for incident visible light $(2.3 \%)$ and have been effectively used for sterilization and heat production. Graphene and its derivatives can be used in the fabrication of masks, PPE and other medical equipment owing to their superhydrophobicity and enhanced light absorption properties [63]. Directa Plus PLC has been claimed to develop masks using nanotechnology based on microbe static, 
antiviral and non-toxic properties of graphene and its derivatives [64]. LIGC Applications has also developed a face mask "Guardian G-Volt" based on laser-induced microporous graphene. It conducts electrical charge to kill the trapped microbes in the filter of mask effectively blocking $99 \%$ contaminants having a size of more than $0.3 \mu \mathrm{m}$ [65].

\section{Nanocomposite-based masks and PPE kits}

A nanocomposite is a composite material possessing at least one of the dimensions of a component in a nanometric scale $(<100 \mathrm{~nm})$. Nanocomposites can be classified as into three categories based on their morphology including phaseseparated systems, intercalated systems and exfoliated systems [14-16]. Various nanocomposite materials have been reported to use in fabrication of masks and PPE [14-16, 29, 63]. Copper3D has claimed to develop an antimicrobial and antiviral face mask "Nanohack" possessing three filtration and disinfecting layers including a non-woven polypropylene impregnated with 5\% copper oxide nanoparticles [66]. A silver nanoparticle-functionalized graphene oxide ink has been developed by ZEN Graphene Solutions Ltd [29]. This viricidal graphene-based composite ink can be employed in N95 face masks and PPE for enhanced protection from COVID19. Czech nanofibre technology firm Respilon Group has developed masks based on three-layer nanofibre membrane with $99.9 \%$ filtration efficiency [67]. The materials used are nanocomposite of copper dioxide $(\mathrm{CuO})$ nanoparticles into the nanofibre matrix.

\section{Air purifiers and filters}

The use of air filters and purifiers has been speculated to reduce the viral load in the environment. Air purifiers can be used in the patient rooms which may reduce the probability of infection of healthcare worker through inadequacies in PPE [68]. It may decrease the possibility of reinfection in a patient due to airborne transmission of viruses. Such filtration system may also be deployed in public transit vehicles, hospital environment, any sites of the aerosol-generating procedure, closed vehicles and home. The size of the fluid droplets of an infected person in cough or sneeze is typically 5 microns or larger [68]. The smallest particle of concern is of the single virion (unattached to any fluid droplet) possessing a diameter of approximately 0.12 microns. The smallest particle that might be of concern is a single virion (unattached to any fluid droplet), having a diameter of approximately 0.12 microns. These may be conceivably filterable by a HEPA (high-efficiency particulate air) filter [68]. ULPA (ultra-low-penetration air) filters are more enhanced in trapping almost $99.99 \%$ of particles 0.12 microns and above [68]. The use of nanotechnology further increases the virus capturing capacity and purification of such air purifiers and filters. Ren et al. [69] have fabricated an efficient filter based on nickel $(\mathrm{Ni})$ foam to catch and kill airborne transmitted viruses and microbes including SARS-CoV-2 and Bacillus anthracis. Since the SARS-CoV-2 virus cannot sustain in temperature above $70{ }^{\circ} \mathrm{C}$, the air filter is designed to work at $200{ }^{\circ} \mathrm{C}$ by heating Ni-foam [69]. The efficiency of the designed filter is claimed to be $99.8 \%$ for SARS-CoV-2 virus and $99.9 \%$ for Bacillus anthracis [69].

\section{Nanofibre technology}

Mack Antonoff HVAC has designed air purification and filtration systems using nanofibrous technology and UV irradiation to combat from COVID-19 [29]. Turn-Key Environmental Consultants have developed an air filtration system based on a dense network of nanofibres (IQAir HyperHEPA® filtration technology), which traps the contaminant particles of all size. It is claimed to capture $99.5 \%$ of contaminants including bacteria and virus of size approximately 0.003 microns [29].

\section{Photoelectrochemical oxidation Technology}

Researchers from the University of South Florida have developed an air purification device "Molekule", which has been claimed to effectively destroy air contaminants including bacteria, mould spores and viruses [70]. The air purifier uses photoelectrochemical oxidation (PECO), in which UV-A light is used to activate a catalyst in the nanoparticle-covered filter to produce free radicals that oxidize air contaminants [70]. These PECO-based air purifiers possess enormous potential to slow down the spread of the virus, predominantly at healthcare facilities.

\section{Immunity modulators}

SARS-CoV-2 infection has a range of presentations and severity in patients. The individuals who are elderly or adults living with comorbidities are more vulnerable to severe outcomes with high rates of morbidity and mortality [23, 29, $71,72]$. Though the correlation of immunity with the risk of infection and severity of its outcomes has not been established yet, it is imperative to mention that individuals with good immunity are protected from the negative outcomes of the disease. Modulation of the immune system has been the base of promising therapies against various severe diseases $[23,29,71,72]$. The composition and physicochemical characteristics of nanocarriers have huge potential to boost immune systems. This aspect of immunomodulation can be achieved either through activation of the immune system against a specific antigen or through the development of immunotolerance against immune-active drugs or other antigens. The former can be used to enhance the efficacy 
of vaccines to develop an optimum and prolonged immune response, and the latter can be used as targeted administration of immunomodulatory drugs [23, 29, 71, 72].

The immunostimulatory nature of traditional antigen in a vaccine can be improved through the use of "potentiators" commonly known as "adjuvants" [23, 29, 71, 72]. The nanoscale drug delivery systems can be modified and used as effective adjuvants for vaccines [23, 29, 71, 72]. This immunomodulation has great potential to improve the efficacy of the vaccines under development against COVID-19.

\section{Diagnosis}

Prompt and early diagnosis is critical to prevent the spread of COVID-19, as it essential to isolate infected individuals and quarantine their contacts [14-16]. Various nanotechnology-based diagnostic approaches such as testing, thermal scanning and biosensing for detecting coronavirus or its symptoms have been developed [14-16, 73-83].

\section{Testing}

Certain diagnostic techniques are possessing a different degree of specificity and are presently available based on single/multiple target molecules of SARS-CoV-2 for its detection [73-76]. These diagnostic tests may involve detection/identification of various aspects including pathological changes in organs of the patient (e.g. computed tomography (CT) scan), viral nucleic acid using one or more gene. (e.g. RT-PCR or reverse transcription polymerase chain reaction), genome or immunological molecules produced by patient or virus in the patient's body (e.g. NGS or next-generation sequencing) and an antigen-antibody reaction (e.g. ELISA test) [14-16, 73-76]. Though the antigen/antibody tests are easy to perform and may give result in 4-6 h and RT-PCR is labour-intensive and gives result in 3-4 h, RT-PCR is the gold standard in the diagnosis of COVID-19 due to its higher sensitivity and specificity [73-76]. However, these tests generally possess shortcomings including long response time, false negative results and poor analytical sensitivity [23, $29,30]$. To address this, nanoparticles with a large surfaceto-volume ratio and high porosity have been used, which allows rapid sensing and reliable detection results [17, 23, 29, 30]. Accordingly, the researchers from University of Maryland, USA, have developed colorimetric sensor based on gold nanoparticles capped with thiol-modified DNA antisense oligonucleotides specific to N-gene (nucleocapsid phosphoprotein) of SARS-CoV-2, which has been used to detect COVID-19 positive cases within $10 \mathrm{~min}$ [30]. The Norwegian University of Science and Technology (NTNU) has developed a magnetic nanoparticle-based detection test using silica-coated iron oxide nanoparticles to extract RNA from the sample of a patient [29, 73-76]. This technology has promised to significantly increase the testing capacity of the laboratories. The Sona Nanotech, Mologic and Sure Screen Diagnostics are developing a quick response lateral flow test through the integration of nanorod technology into a disposable lateral flow platform. This test has the potential to screen patients in 5-15 min [29]. However, the quantity of viral load used is one of the major shortcomings in such tests. Contrary to this, paper-nanomaterial-based sensors are cost-effective devices to diagnose COVID-19-related biomarkers [23]. These devices are popular for providing rapid results which can be observed through naked eyes or can be analysed using smartphones [23]. A paper-based colorimetric test to detect DNA of MERS-CoV, Mycobacterium tuberculosis and human papillomavirus using pyrrolidinyl peptide nucleic acid (acpcPNA)-induced silver nanoparticle aggregation possesses the potential for the detection of SARS-CoV-2 [30].

\section{Thermal scanning}

Thermal scanners have been vastly used as one of the detection techniques for monitoring COVID-19 symptomatic patients [14-16, 29, 77]. It is important to mention that these thermal scanners can only detect elevated skin temperatures, which is quite different from core body temperature. Therefore, they are not precise to determine whether the individual possesses a fever or not. People with heavy builds or hot flushes and under various temporary environmental conditions (e. g., walking in from a hot car) can possess elevated skin temperature, which will trigger the alarm of such thermal scanners. Moreover, fever is not one of the common symptoms in almost $75 \%$ of COVID-19 patients [4, 14-16]. Thus, the thermal scanning as a preventive measure is a vague idea. However, it is important to check the temperature of every individual as a precautionary measure at crowded places including hospitals, malls, offices and markets to have a primary idea about the presence of a sick individual. The temperature detection efficiency and limit of detection for such thermal scanning equipment can be enhanced using nanotechnology [14-16, 77]. Various nanomaterials have been reported to use nanomaterials for the fabrication of probe of thermal scanners [14-16, 77].

\section{Biosensors}

Biosensors are autonomous integrated monitoring device comprising of a bioreceptor, a transducer and a signal detector [78]. The interaction amongst bioreceptor and target analyte including microbes generate an electrical signal, which is further amplified using transducers and finally detected and displayed by a signal detector. Advanced biosensor-based diagnosis approaches use nanomaterials with 
tunnelling and quantum properties resulting in enhanced signal amplification from the target analytes (viruses) [78] These nanomaterials also have a high surface-to-volume ratio which results in the enhancement of their sensitivity. As viruses measure in nanoscale, these features of nanosensors make them potential diagnostic tools. This class of testing is also known as advanced POCT (point of care tests) approaches [78, 79]. These tests can be used without the presence of an expert or trained person at the patient's bedside. Various types of nano-biosensors can be employed in handheld POCT diagnostic techniques to detect SARSCoV-2 with high sensitivity, selectivity and accuracy as shown in Fig. 3 [78, 79].

\section{Aptamer-based nano-biosensor}

Aptamer-based nano-biosensor technique is one of the most potent monitoring tools for rapid diagnosis of various disease-causing microbes with high sensitivity and selectivity. Contrary to conventional diagnostic techniques, this is a very cost-effective, user- and eco-friendly technique. Aptamers can be specifically fabricated for any virus using its nucleocapsid protein possessing a high specific binding affinity for specific target molecules [78, 79]. The target molecule can be any component of virus including amino acid, membrane protein, cytokines, toxins, coupling agents or immunoglobulins $[78,79]$. These fabricated aptamers use apta-sensors, which can be easily transformed to any specific required design through chemical modification or surface activation to induce coupling sites and linkers [79]. These bio-nanogate bifunctional aptamer-based sensor works through electrochemical measurements (Fig. 4) [79]. Therefore, aptamerbased nanosensor will have great potential for the detection of SARS-CoV-2 even in person without any symptoms with rapid results, high sensitivity, specificity and selectivity only for COVID-19 [80, -81]. Pinpoint's aptamer-based biosensors for monitoring SARS-CoV-2 are reported to be under trial $[78,79]$. Aptamer for the said biosensors has been synthesized from SARS-CoV-2 nucleocapsid spike S1 protein, which makes it unique from other viruses. The viral agent has been taken from a nasal or throat swab sample of an individual [79]. This bio-nanogate bifunctional apta-sensor specifically responds to SARS-CoV-2 nucleocapsid spike $\mathrm{S} 1$ protein as a target molecule, and the response is measured through electrochemical analysis [78, 79]. It has been claimed to provide SARS-CoV-2 test result within $1 \mathrm{~min}$ and to be precise in only $30 \mathrm{~s}[78,79]$.

\section{Plasmonic photothermal dual-functional biosensors}

Dual-functional plasmonic photothermal biosensors are the combination of two techniques including the plasmonic photothermal (PPT) effect and localized surface plasmon resonance (LSPR) effects. Wang et al. have reported designing a dual-functional biosensor based on similar techniques [80]. They have used DNA receptors as a detector which can monitor the selective sequences of SARS-CoV-2 using nucleic acid hybridization. The sensing performance has
Fig. 3 Various bio-sensing techniques for the detection of SARS-CoV-2

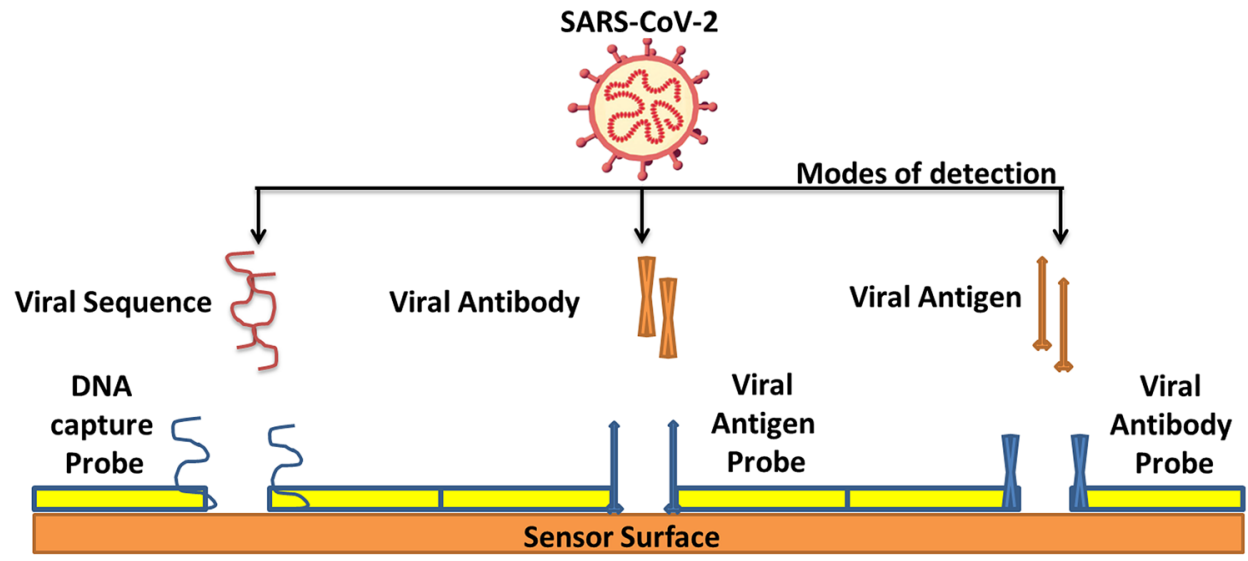

Immobilization

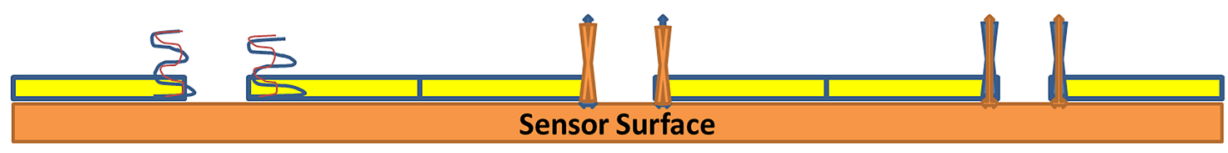

Signal generation 
Fig. 4 Working of aptamerbased biosensor

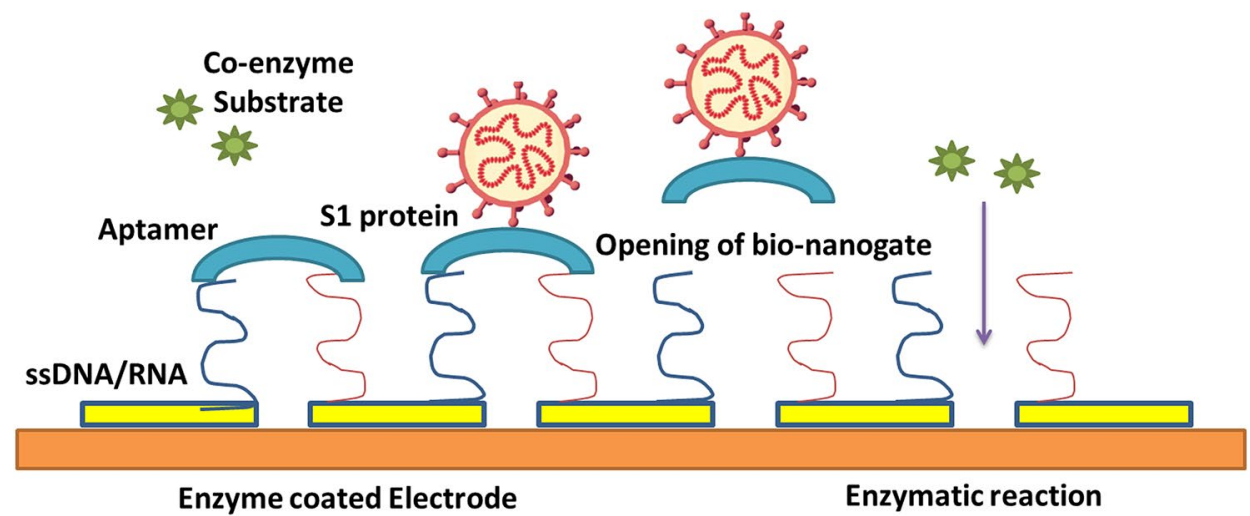

been claimed to be enhanced using nanogold-based chip (AuNIs). On illumination to its plasmonic resonance frequency, AuNIs chips generate the thermoplastic heat. This generated localized PPE heat elevates in situ hybridization temperature and expedite the precise differentiation of two similar gene sequences. It has been claimed to possess very low detection limit as low as $0.22 \mathrm{pM}$ of concentration, high sensitivity and high selectivity for precise detection of a specific gene through the multigene mixture [80].

\section{Graphene-based biosensor}

These types of sensors are fabricated by coating graphene sheets with a selective antibody against SARS-CoV-2 spike protein G. Seo et al. [81] have reported a graphene FET device for the detection of the SARS-CoV-2 spike protein at concentrations of $1 \mathrm{fg} / \mathrm{mL}$ in phosphate-buffered saline and $100 \mathrm{fg} / \mathrm{mL}$ clinical transports medium. The detection of the virus in the air has also been claimed in this report [81]. Groltex and Sanford Burnham Prebys Medical Discovery Institute has claimed to develop a graphene-based biosensor platform consisting of handheld reader units and disposable plastic testing chips [82]. Layqah et al. [850] have also reported an electrochemical immunosensor using modified carbon electrodes with gold nanoparticles for the monitoring of human coronavirus ( $\mathrm{HCoV}$ ) and MERS-CoV proteins in spiked nasal samples. Such graphene-based sensors possess enormous potential to detect SARS-CoV-2 [78-83].

\section{Treatment}

\section{Medicines and drug delivery agents}

Various pharmaceuticals are currently involved in the development and evaluation of promising molecules that have the potential to treat COVID-19 patients [84-87]. Nanotechnology has been effectively used in the development of treatment of cardiovascular, gastrointestinal and neurological disorders, cancers and HIV/AIDS [84-87]. Medicines made with nanoparticles have great advantages as nanoparticles can improve their solubility, and increase the resistance to acid and enzymes present in stomach and intestine leading to their better absorption in the intestine [84-87]. They are also effective for controlled and sustained release of drugs in the body leading to a maintained level of drugs in the body tissue. A wide range of active therapeutic agents: antivirals, biologics and nucleic acids can be loaded and delivered by nanocarriers. Bioavanta LLC has developed a safe and effective drug delivery technology which uses biocompatible chitosan-based nanoparticle aerosol formulation named NovochizolTM [85]. These nanoparticles strongly adhere to lung epithelial tissues and can act as an ideal intra-pulmonary drug delivery system for effective drugs. Researchers at MIT have discovered a peptide molecule that strongly and specifically binds with the spike protein of coronavirus [86]. However, they have limited efficacy as enzymes in our body can rapidly degrade this molecule. The researchers at Northwestern Simpson Querrey Institute (SQI) are working on the development of peptide-based nanostructures that can act as a carrier of precious drugs, thus protecting it while circulating in the body before it encounters the virus [87].

The nanomedicine approach of reformulating approved as well under trial drug candidates to improve their therapeutic index (TI) involves connecting the right therapeutic candidate with the right nanocarrier [88]. This approach is crucial for the success of nanomedicine against SARS-CoV-2 [88]. The proposed strategies to fast-track COVID-19 nanomedicine research are:

Strategy 1: Nanocarrier selection to bypass the conventional limitations of a drug candidate.

Nanocarriers are used to prevent the systemic immunotoxicity of the protein-based drugs [89]. For example, lipid nanoparticles (LNPs) carrying siRNA are an example of a nanotechnology platform used to prevent systemic degradation of siRNA along with the benefit of liver-targeting [27].

Strategy 2: Chemically alter/(re)engineer drugs. 
Drug molecules are altered or re-engineered to improve their binding with a particular class of nanocarriers leading to a more generic approach for drug candidates [90]. For example, lipid bilayer nanocarriers are preferred for $\mathrm{PH}$ gradient-based remote loading of amphiphilic and ionizable drugs [90].

Strategy 3: Nanomedicine for combination drug therapeutics.

This approach involved the delivery of multiple drugs with different physicochemical properties through nanocarriers [27, 88-90]. It has the advantage of delivery of lower dosage of individual drugs resulting in better efficacy and fewer side effects, achieving multiple and therapeutic targets and reducing the chances of development of resistance [88]. Some of these combinations have been documented in WHO landscape information (Table 3) [88]. However, till now no drug has been approved for the treatment of COVID-19.

\section{Ventilators}

The use of various antiviral and antimicrobial nanomaterial (such as silver, gold nanoparticles, graphene)-based coating on ventilator units can give rise to a new class of ventilators based on nanotechnology [91]. The research and development of such nanomaterial-based antiviral coatings has been already discussed in the above section. The inclusion of such coatings on ventilators will reduce the possibility of infection and cross-infections from bacteria, virus and other microbes [14-16, 39-45, 91].

\section{Medical treatments for symptoms}

So far, no evident treatment or vaccine has been registered for the treatment of COVID-19 except for some conventionally used medication to improve the immune system and provide relief symptomatically $[92,93]$. However, the interesting and surprising properties of chemical compounds, especially nanodrugs, can significantly contribute not only to the medicine and pharmaceutics, but they can also emerge as promising solutions to stop the deadly COVID-19 outbreak across the world $[92,93]$.

\section{Radiation-based treatment}

Photobiomodulation (PBM) is a photon therapy that uses photons from light-emitting diodes, LASER and broadband light, in the visible and near-infrared range [94, 95]. Depending upon the intensity and wavelength of a light source, these therapies are used to target various tissues of the human body including transcranial brain and bloodstream. A therapy based on LASER with high penetrating power used for treatment procedures in the thoracic cavity is known as transthoracic PBM therapy $[94,95]$. This therapy is similar to infrared LASER-based therapies of 810 or $940-970 \mathrm{~nm}$, which can penetrate the skull tissue. Such treatments are also effective for diseases related to lungs and other diseases including tuberculosis, pneumonia or influenza. This nanotechnology-based technique has potential for the treatment of COVID-19, but research is still under exploration $[94,95]$.

\section{Conclusions}

The COVID-19 pandemic has led to an unprecedented loss of lives and economy across the world. Still, the spread of the virus is not stopping and the number of cases and mortality due to it is increasing daily. The need for this situation is the collaboration of scientists, physicians and engineers to combat this global threat of COVID-19 and to custom a strong response to tackle such pandemics in future. There is an urgent need for the global response to commit funding and strengthen research and development in science and technology with interdisciplinary collaborations. Nanotechnology has been used to enhance the present-day tools and techniques to combat such pandemic situations. It has huge potential for breakthrough innovations for future advancement of tools to prevent, diagnose and treat various emerging and remerging diseases. The review illustrates the immense research and development in the field of nanotechnology to combat COVID-19 pandemic. The world is growing fast to effectively fight against the novel virus, but there is a strong
Table 3 Status of candidate of medicines for COVID-19 [27, 88-90]

\begin{tabular}{lll}
\hline Combination description & Candidate drugs & Status \\
\hline Protease inhibitors & Lopinavir + ritonavir & $\begin{array}{c}\text { Not effective, the solidar- } \\
\text { ity trial has stopped this } \\
\text { arm }\end{array}$ \\
$\begin{array}{l}\text { Non-nucleoside reverse transcriptase } \\
\text { inhibitor + nucleoside reverse tran- } \\
\text { scriptase inhibitor }\end{array}$ & Emtricitabine + tenofovir & Under trial \\
$\begin{array}{l}\text { Anti retroviral protease inhibitor+cobi- } \\
\text { cistat (to improve bioavailability and } \\
\text { t half) }\end{array}$ & Darunavir + cobicistat & Under trial \\
\hline
\end{tabular}


need for global commitment to support research and development in this field.

Acknowledgement The authors wish to acknowledge the Vice-Chancellor of the University of Delhi and Department of Science and Technology, Government of India, for providing the e-resources.

\section{References}

1. World Health Organization: Coronavirus Disease (COVID-19), Pandemic, Accessed 23032020 from https://www.who.int/emerg encies/diseases/novelcoronavirus- 2019

2. Shereen MA et al (2020) COVID-19 infection: origin, transmission, and characteristics of human coronaviruses. J Adv Res 24:91-98

3. World Health Organization: Coronavirus Disease (COVID-19) Pandemic, https://covid19.who.int/.

4. World Health Organization: Coronavirus Disease (COVID-19) Pandemic, https://www.who.int/news-room/commentaries/detai 1/modes-of-transmission-of-virus-causing-covid-19-implicatio ns-for-ipc-precaution-recommendations).

5. Zhang J et al (2020) Fecal specimen diagnosis 2019 novel coronavirus-infected pneumonia. J Med Virol 92:680-682

6. Gao J et al (2020) Chloroquine phosphate has shown apparent efficacy in treatment of COVID-19 associated pneumonia in clinical studies. Biosci Trends 14:72-73

7. Chang Y-C et al (2020) Potential Therapeutic Agents for COVID19 Based on the Analysis of Protease and RNA Polymerase Docking. Preprints: 2020020242.

8. Colson P et al (2020) Chloroquine and hydroxychloroquine as available weapons to fight COVID-19. Int J Antimicrob Agents 55:105932

9. Lai C-C et al (2020) Severe acute respiratory syndrome coronavirus 2 (SARS-CoV-2) and coronavirus disease-2019 (COVID19): The epidemic and the challenges. Int J Antimicrob Agents 55:105924

10. World Health Organization: Coronavirus Disease (COVID-19) Pandemic, https://www.who.int/teams/blueprint/covid-19

11. Del Rio C et al (2020) COVID-19 - new insights on a rapidly changing epidemic. JAMA 323(14):1339-1340

12. Mei $X$ et al (2020) Artificial intelligence-enabled rapid diagnosis of patients with COVID-19. Nat Med: https://doi.org/https://doi. org/10.1038/s41591-020-0931-3

13. Chung $\mathrm{M}$ et al (2020) CT imaging features of 2019 novel coronavirus (2019-nCoV). Radiology 295:202-207

14. Sportelli MC et al (2020) Can nanotechnology and materials science help the fight against SARS-CoV-2? Nanomaterials 10(4):802

15. Kalantar-Zadeh $\mathrm{K}$ et al (2020) Considering the effects of microbiome and diet SARS-COV-2 infection: nanotechnology roles. ACS Nano 14(5):5179-5182

16. Chan WCW et al (2020) Nano Research for COVID-19. ACS Nano 14(4):3719-3720

17. Bhushan B (2007) Springer handbook of nanotechnology. Springer, Germany

18. Nasrollahzadeh $\mathrm{M}$ et al (2020) Nanomaterials and nanotechnology-associated innovations against viral infections with a focus on coronaviruses. Nanomaterials 10:1072

19. Chaudhary V et al (2016) Surfactant directed polyaniline nanostructures for high-performance sulphur dioxide chemiresistors: effect of morphologies, chemical structure and porosity. RSC Adv 6:95349-95357
20. Chaudhary V et al (2015) Enhanced and selective ammonia sensing behaviour of poly(aniline co-pyrrole) nanospheres chemically oxidative polymerized at low temperature. JIEC 26:143-148

21. Mokhtarzadeh A et al (2017) Nanomaterial based biosensors for detection of the pathogenic virus. TrAC Trends Anal Chem 97:445-457

22. Palestino G et al (2020) Can nanotechnology help in the fight against COVID-19? Expert Rev Anti-infective Therapy 18:849864. https://doi.org/10.1080/14787210.2020.1776115

23. Huang $\mathrm{C}$ et al (2020) Clinical features of patients infected with 2019 novel coronavirus in Wuhan. China Lancet 395(10223):497-506

24. Weiss $\mathrm{C}$ et al (2020) Toward nanotechnology-enabled approaches against the COVID-19 pandemic. ACS Nano 14:6383-6406

25. Nikaeen G, Abbaszadeh S, Yousefinejad S (2020) Application of nanomaterials in treatment, anti-infection and detection of coronaviruses. Nanomedicine 15(15):1501-1512

26. WHO:https://www.who.int/publications/m/item/draft-landscapeof-covid-19-candidate-vaccines.

27. Chauhan $G$ et al (2020) Nanotechnology for COVID-19: therapeutics and vaccine research. ACS Nano 14(7):7760-7782

28. Lawrence $C$ et al (2020) A strategic approach to COVID-19 vaccine R\&D. Science 368(6494):948-950

29. Look M et al (2010) Application of nanotechnologies for the improved immune response against infectious diseases in the developing world. Adv Drug Deliv Rev 62(4-5):378-393

30. https://statnano.com/technology-against-covid-19-nano-insights.

31. Vaze $\mathrm{N}$ et al (2019) Inactivation of common hospital-acquired pathogens on surfaces and in air utilizing engineered water nanostructures (EWNS) based nano-sanitizers. nanomedicine: nanotechnology. Biolo Med 18:234-242

32. Deshmukh SP et al (2019) Silver nanoparticles as an effective disinfectant: a review. Mater Sci Eng: C 97:954-965

33. Dizaj SM et al (2014) Antimicrobial activity of the metals and metal oxide nanoparticles. Mater Sci Eng: C 44:278-284

34. Nakamura S et al (2019) Synthesis and application of silver nanoparticles (Ag NPs) for the prevention of infection in healthcare workers. Int J Mol Sci 20:3620

35. Nature India (2020): doi:https://doi.org/10.1038/nindia.2020.62 Published online 6 April 2020: https://www.natureasia.com/en/ nindia/article/https://doi.org/10.1038/nindia.2020.62.

36. Silva LP (2019) Green Nanotechnology for Sustained Release of Eco-Friendly Agrochemicals. In: Vaz Jr S (eds) Sustainable Agrochemistry Springer, Cham

37. Ramkumar P et al (2020) Times of India May 14, 2020: http:// timesofindia.indiatimes.com/articleshow/75726359.cms?utm source $=$ contentofinterest\&utm_medium $=$ text\&utm_campa ign $=$ cppst.

38. PIB Mumbai, 08 JUN 2020: https://pib.gov.in/PressReleasePag e.aspx?PRID=1630197.

39. Park YJ et al (2019) Guanidine-based disinfectants, polyhexamethylene guanidine-phosphate (PHMG-P), polyhexamethylene biguanide (PHMB), and oligo(2-(2-ethoxy)ethoxyethyl guanidinium chloride $(\mathrm{PGH})$ induced epithelial-mesenchymal transition in A549 alveolar epithelial cells. Inhalation Toxicol 31(4):161-166

40. Expats $\mathrm{C} \mathrm{Z}$ et al (2020) Antiviral and antibacterial coatings being tested on Prague trams and buses: https://news.expats.cz/healt h-medical/antiviral-and-antibacterial-coatings-being-tested-onprague-trams-and-buses/.

41. Makvandi P et al (2020) Metal-based nanomaterials in biomedical applications: antimicrobial activity and cytotoxicity aspects. Adv Funct Mater 30:1910021

42. https://www.nano4life.co/nano4-hygienen-life.

43. Montazer M et al (2020) Nanotoxicity: prevention and antibacterial applications of nanomaterials. Micro and Nano Technol 
202:399-413. https://doi.org/10.1016/B978-0-12-819943-5.00020 $-8$

44. Martínez L, Kharissova O, Kharisov B (eds) (2018) Handbook of ecomaterials. Springer, Cham. https://doi.org/10.1007/978-3-31948281-1

45. Hwang GB et al (2020) Photobactericidal activity activated by thiolated gold nanoclusters at low flux levels of white light. Nat Commun 11:1207

46. NanoSeptic Continuously Self-Cleaning Surfaces; NanoSeptic${ }^{\circledR N}$ NanoTouch and ${ }^{\circledR}$ NanoSeptic are the registered trademarks of NanoTouch Materials; https://www.nanoseptic.com.

47. Ahani E et al (2017) A novel biocompatible antibacterial product: Nanoliposomes loaded with poly(hexamethylene biguanide chloride). J Bioact and Compat Polym 32(3):242-262

48. https://medicalxpress.com/news/2020-05-technology-powerfuldisinfectant-viruses.htm.

49. Punter Villagrasa $\mathbf{J}$ et al (2017) Amperometric and impedance monitoring systems for biomedical applications. Springer, Germany

50. Konda A et al (2020) Aerosol filtration efficiency of common fabrics used in respiratory cloth masks. ACS Nano 14(5):6339-6347

51. Mahapatra PS, Chatterjee S, Tiwari MK et al (2020) Surface treatments to enhance the functionality of PPEs. Trans Indian Natl Acad Eng 5:333-336. https://doi.org/10.1007/s41403-020-00110 $-0$

52. PIB Delhi: Release ID: 1617541: 23/04/2020: https://pib.gov.in/ PressReleasePage.aspx?PRID $=1617541$.

53. Nature India: doi:https://doi.org/10.1038/nindia.2020.65 Published online 15 April 2020: Antimicrobial spray coat against coronavirus: https://www.natureasia.com/en/nindia/article/https ://doi.org/10.1038/nindia.2020.65.

54. Promethean Particles: Apr 14, 2020: https://www.prometheanparti cles.co.uk/promethean-particles-puts-its-nano-copper-to-the-testin-the-fight-against-viruses/.

55. Sonovia's SonoMask ${ }^{\mathrm{TM}}$ have been proven to filtrate $98 \%$ of 5 microns particles, in accordance with WHO regulation. https:// sonoviatech.com/

56. Photonics Spectra, June 2020: https://www.photonics.com/Artic les/Coating_That_Could_Destroy_COVID-19_Virus_for/a65712.

57. New World HK, Press Release: 28 February 2020: https://www. nwd.com.hk/content/new-world-\%E2\%80\%99s-adrian-cheng -announces-new-initiatives-combat-coronavirus-first-corpo rate-i-0.

58. Recyclable nano-filter for face masks developed (2020). https ://www.labonline.com.au/content/consumables/news/recyclable -nano-filter-for-face-masks-developed-341058879

59. Queensland University of Technology (QUT): Press Release dated 15 April 2020: https://www.qut.edu.au/news?id=161468.

60. Korea Advanced Institute of Science and Technology: News dated 17 March 2020: http://news.kaist.ac.kr/newsen/html/ news $/$ mode $=V \&$ mng_no $=6530$.

61. Yamashin Filter co.: http://www.yamashin-filter.co.jp/eng/nano. html.

62. https://amritaworld.org/education/low-cost-amrita-nano-masks -to-prevent-coronavirus-spread/: $5^{\text {th }}$ May 2020.

63. Metamasks filters feature an exclusive New Zealand nanofibre tested by NELSON LABS in June 2020. https://www.metamasks. com/nanotech

64. https://www.avalon-nanofiber.com/product/nanopoly-nanof iber-mask/.

65. Palmieri V et al (2020) Can graphene take part in the fight against COVID-19? Nanotoday 33:100883

66. Directa Plus PLC: John Harrington, 23 March 2020: https://www. proactiveinvestors.com/companies/news/915486/directa-plushighlights-bacteriostatic-properties-of-its-graphene-915486.html.
67. Deezen: India Block: 6 March 2020: https://www.dezee n.com/2020/03/06/guardian-g-volt-face-mask-graphene-coron avirus-bacteria/.

68. About Nanohack 2.0. https://copper3d.com/hackthepandemic /\#About_NanoHack\%E2\%80\%8E

69. Czech Trade: 04 Feb, 2020: https://www.czechtradeoffices.com/ en/id/news/czech-company-respilon-develops-_revolutionary_facemask-helping-against-coronavirus.

70. Elias B et al (2020) Could air filtration reduce COVID-19 severity and spread?. New England Complex Systems Institute (March 9, 2020).

71. Molekule's patented PECO technology. https://molekule.com/ technology

72. Dacoba TG et al (2017) Modulating the immune system through nanotechnology. Semin Immunol 34:78-102

73. Hammou RA (2020) Chapter Application of Nanodiagnostics in Viral Infectious Diseases. In: M Mustapha Ennaji (ed) Emerging and Reemerging Viral Pathogens, Academic Press, US

74. Muhammad Q et al (2020) Modulation of immune responses with nanoparticles and reduction of their immunotoxicity. Biomater Sci 8:1490-1501

75. Kumar R et al (2020) COVID-19 diagnostic approaches: different roads to the same destination. Virus Dis. (2020). https://doi. org/https://doi.org/10.1007/s13337-020-00599-7

76. Vafea $\mathrm{T}$ et al (2020) Emerging technologies for use in the study, diagnosis, and treatment of patients with COVID-19. Mol Bioeng Cel 13:249-257. https://doi.org/10.1007/s12195-020-00629-w

77. https://cordis.europa.eu/article/id/415876-molecular-mechanisms -and-methods-used-to-detect-covid-19-infection.

78. Tang YW et al (2020) Laboratory diagnosis of COVID-19: current issues and challenges. J Clin Microbiol 58(6):0051220

79. Shin J et al (2020) Sensitive wearable temperature sensor with seamless monolithic integration. Adv Mater 32:1905527

80. Christopher FC et al (2020) Recent advancements in rapid analysis of pesticides using nano biosensors: a present and future perspective. J Clean Prod 269:122356

81. Samson R et al (2020) Biosensors: frontiers in rapid detection of COVID-19. Biotech 10:385

82. Qiu G et al (2020) Dual-functional plasmonic photothermal biosensors for highly accurate severe acute respiratory syndrome coronavirus 2 detection. ACS Nano 14(5):5268-5277

83. Seo $\mathrm{G}$ et al (2020) rapid detection of covid-19 causative virus (sars-cov-2) in human nasopharyngeal swab specimens using fieldeffect transistor-based biosensor. ACS Nano 14(4):5135-5142

84. Graphene-info: 21 April, 2020: Grolltex develops graphene-based sensor for Covid-19 detection: https://www.graphene-info.com/ grolltex-develops-graphene-based-sensor-covid-19-detection

85. Layqah LA et al (2019) An electrochemical immunosensor for the corona virus associated with the Middle East respiratory syndrome using an array of gold nanoparticle-modified carbon electrodes. Microchim Acta 186(4):224

86. Kumar $\mathrm{N}$ et al (2013) Nanotechnology and Nanomaterials in the Treatment of Life-threatening Diseases: 1st Edition, ISBN: 9780323264334, Imprint: William Andrew, Published Date: 16th December 2013, Elsevier.

87. Novochizol ${ }^{\mathrm{TM}}$ is a first-in-class nanoparticle generation technology. https://www.novochizol.ch/what/

88. Bioavanta-Bosti: Chitosan nanoparticles suitable for aerosol treatment of Covid-19 patients. https://www.swissbiotech.org/listing/ bioavanta-bosti-announces-immediate-availability-of-its-chito san-nanoparticle-technology-to-formulate-aerosol-anit-covid -19-drugs/

89. Chakravarty M, Vora A (2020) Nanotechnology-based antiviral therapeutics. Drug Deliv Transl Res. https://doi.org/10.1007/ s13346-020-00818-0 
90. Zhang $\mathrm{Y}$ et al (2018) Nanoparticle anchoring targets immune agonists to tumors enabling anti-cancer immunity without systemic toxicity. Nat Commun 9(1):6

91. Adams D et al (2018) An RNAi therapeutic, for hereditary transthyretin amyloidosis. N Engl J Med 5379(1):11-21

92. Cern A et al (2017) New drug candidates for liposomal delivery identified by computer modeling of liposomes' remote loading and leakage. J Control Release 28(252):18-27

93. Zachar $\mathrm{O}$ et al (2020) Formulations for COVID-19 Treatment via Silver Nanoparticles Inhalation Delivery: Preprint: June 2020: DOI: https://doi.org/10.31219/osf.io/adnyb: https://www.resea rchgate.net/publication/340270205_Formulations_for_COVID -19_Treatment_via_Silver_Nanoparticles_Inhalation_Delivery.

94. COVID-19 and Nanotechnology - Nanografi Blog, https://nanog rafi.com/blog/covid19-and-nanotechnology/.

95. Christian A et al (2020) New insights on the antiviral effects of chloroquine against coronavirus: what to expect for COVID-19? Int J Antimicrob Agents 55(5):105938
96. Anders J (2019) Light-emitting diode therapy and low-level light therapy are photobiomodulation therapy. Photobiomodulation Photomed Laser Surg 37(2):63-65

97. Fekrazad R et al (2020) Photodynamic therapy with phthalomethyl D: perspectives against SARS-CoV-2. Photobiomodulation, Photomed, Laser Surg 38(5):255-257

98. Almeida A et al (2020) Antimicrobial photodynamic therapy in the control of COVID-19. Antibiotics 9:320

Publisher's Note Springer Nature remains neutral with regard to jurisdictional claims in published maps and institutional affiliations. 